\title{
Index nominum ad Vol. 162
}

Aeppli, H., 311 (S)

Aeppli, L., 311 (S)

Arnold, M., 447 (S)

Barna, K., 203

Barzilai, D., 216

Beeguer, J., 398 (S)

Béguin, F., 439 (S)

Berger, J., 301 (S), 316 (S)

Bloch, S., 105, 261

Boczowski, K., 69

Bossart, H., 360 (S)

Bretscher, J., 369 (S)

Brun, G., 254

Buess, H., 453

Cavegn, B., 341 (S)

Chomicki, O., 206

Cloeren, St., 447 (S)

Dubecq, J. P., 254

Ebner, R., 1, 386 (S)

Eckerling, B., 225

Ehrler, P., 104 (B)

Emmrich, P., 241

Engelhorn, A., 350 (S)

Erb, H., 275

Fassolt, A., 320 (S)

Gábor, M., 283

Gamp, R., 1, 386 (S)

Gaudyn, J., 206

Gavallér, I., 165

Geisendorf, W., 398 (S), 445 (S)

Glatthaar, E., 335 (S)

Górowski, T., 206

Gömöri, Z., 79

Hackl, H., 233

Hauser, G. A., 169,320 (S), 327 (S), 387 (S)

Hawrylenko, A., 1, 384 (S)

Hedberg, G., 213

Hinselmann, M., 419 (S)

Hüter, K. A., 57 
Jaroszewicz, B., 206

Jirâsek, J. E., 147

Keller, P. J., 402 (S)

Roller jr., Th., 1, 384 (S)

Koltoniak, D., 48

Krieg, H., 156

Léderrey, P., 398 (S)

Lutzinger, H., 380 (S)

Madej, J., 119

Mahon, R., 254

Mall-Haefeli, M., 447 (S)

Mattern, L., 350 (S)

Mestral, A. de, 320 (S)

Meudt, R., 430 (S)

Meyer, B., 125

Meylan, J., 445 (S)

Morf, E., 447 (S)

Naville, A. H., 402 (S)

Neri, A., 225

Neuweiler, W., 308 (S), 380 (S)

Numers, C. von, 125

Obolensky, W., 175

Onwudiwe, E. U., 308 (S)

Ott, F., 387 (S)

Paldi, E., 216

Papadopoulos, S., 360 (S)

Piukovich, I., 283

512

Index nominum ad Vol. 162

Pokorný, J., 185

Rautalinko, T., 88

Rehsteiner, H. P., 299 (S), 414 (S)

Richter, R. H. H., 447 (S)

Riotton, G., 398 (S)

Rippmann, E. T., 316 (S)

Rosenblatt, R., 350 (S)

Roth, F., 447 (S)

Ruffieux, M., 350 (S)

Sabeti, S., 350 (S)

Saling, E., 505 (B)

Salvatore, C. A., 95

Schlegel, L., 185

Schlemmer-Perlitz, I., 311 (S)

Schneider, D., 391 (S)

Schubiger, V., 169 
Schweizer, E., 424 (S)

Simm, S., 48

Spitzbart, H., 139

Staib, F., 156

Stamm, H., 289 (S), 447 (S)

Stamm, O., 341 (S), 439 (S)

S. Stembera, Z. K., 185 Stockhammer, H., 391 (S) Stucki, D., 435 (S) Szöllösy, J., 283 Szontágh, F. E., 283 Teter, J., 69 Timonen, S., 125 Voegeli, H., 398 (S) Volken, B., 327 (S) Vulliémoz, P., 350 (S) Watteville, H. de, 350 (S) Weber, H., 341 (S) Weber, M., 335 (S) Webün, L., 233 Weis, P., 447 (S) Wespi, H. J., 299 (S), 414 (S) Wessela, E., 203 Widholm, O., 88 Wyss, H., 402 (S), 447 (S) Zvarík, E., 197 Zwinger, A., 147 